\title{
Driveline Features as Risk Factor for Infection in Left Ventricular Assist Devices: Meta-Analysis and Experimental Tests
}

\begin{abstract}
Melanie Kranzl ${ }^{1}$, Martin Stoiber ${ }^{2}$, Anne-Kristin Schaefer ${ }^{1}$, Julia Riebandt ${ }^{1}$, Dominik Wiedemann ${ }^{1}$, Christiane Marko ${ }^{1}$, Günther Laufer ${ }^{1,3}$, Daniel Zimpfer ${ }^{1,3}$, Heinrich Schima ${ }^{1,2,3}$ and Thomas Schlöglhofer ${ }^{1,2,3 *}$
\end{abstract}

${ }^{1}$ Department of Cardiac Surgery, Medical University of Vienna, Vienna, Austria, ${ }^{2}$ Center for Medical Physics and Biomedical Engineering, Medical University of Vienna, Vienna, Austria, ${ }^{3}$ Ludwig-Boltzmann-Institute for Cardiovascular Research, Vienna, Austria

\section{OPEN ACCESS}

Edited by:

Michael Lichtenauer,

Paracelsus Medical University, Austria

Reviewed by:

Jens Garbade,

Klinikum Links der Weser, Germany Moritz Mirna,

Paracelsus Medical University, Austria

*Correspondence:

Thomas Schlöglhofer thomas.schloeglhofer@

meduniwien.ac.at

orcid.org/0000-0003-4354-4860

Specialty section

This article was submitted to Heart Failure and Transplantation, a section of the journa

Frontiers in Cardiovascular Medicine

Received: 27 September 2021 Accepted: 08 November 2021 Published: 16 December 2021

Citation:

Kranzl M, Stoiber M, Schaefer A-K, Riebandt J, Wiedemann D, Marko C, Laufer G, Zimpfer D, Schima H and Schlöglhofer T (2021) Driveline Features as Risk Factor for Infection in Left Ventricular Assist Devices: Meta-Analysis and Experimental Tests.

Front. Cardiovasc. Med. 8:784208 doi: 10.3389/fcvm.2021.784208
Background: Risk factors for driveline infection (DLI) in patients with left ventricular assist devices are multifactorial. The aim of this study was to analyze the correlation between mechanical driveline features and DLI occurrence.

Methods: A meta-analysis was conducted that included studies reporting DLI rates at 6 months after implantation of any of three contemporary devices (HVAD with Pellethane or Carbothane driveline, HeartMate II, and HeartMate 3). Further, outer driveline diameter measurements and ex-vivo experimental three-point bending and torsion tests were performed to compare the stiffness of the four different driveline types.

Results: 21 studies with 5,393 patients were included in the meta-analysis. The mean weighted DLI rates ranged from $7.2 \%$ (HeartMate II) to $11.9 \%$ (HeartMate 3). The HeartMate II driveline had a significantly lower maximal bending force (Load $\max$ ) (4.52 $\pm 0.19 \mathrm{~N})$ compared to the Carbothane HVAD $(8.50 \pm 0.08 \mathrm{~N})$, the HeartMate $3(11.08$ $\pm 0.3 \mathrm{~N})$, and the Pellethane HVAD driveline $(15.55 \pm 0.14 \mathrm{~N})(p<0.001)$. The maximal torque (Torque max $_{\text {) }}$ of the HeartMate II [41.44 (12.61) mNm] and the Carbothane HVAD driveline [46.06 (3.78) $\mathrm{mNm}$ ] were significantly lower than Torque $\max _{\text {of }}$ of the Pellethane HVAD [46.06 (3.78) mNm] and the HeartMate 3 [95.63 (26.60) mNm] driveline $(p<$ 0.001). The driveline of the HeartMate 3 had the largest outer diameter [6.60 $(0.58) \mathrm{mm}]$. A relationship between the mean weighted DLI rate and mechanical driveline features (Torque $_{\max }$ ) was found, as the the HeartMate II driveline had the lowest Torque $\max _{\text {ax }}$ and lowest DLI rate, whereas the HeartMate 3 driveline had the highest Torque $\max _{\text {ax }}$ and highest DLI rate.

Conclusions: Device-specific mechanical driveline features are an additional modifiable risk factor for DLI and may influence clinical outcomes of LVAD patients.

Keywords: left ventricular assist device (LVAD), mechanical features, risk factors, driveline infection, mechanical circulatory support (MCS) 


\section{INTRODUCTION}

Heart failure remains among the main causes of morbidity and mortality worldwide with an increasing prevalence $(1,2)$. In recent years, left ventricular assist devices (LVADs) have become an established therapeutic option for end-stage heart failure (3) to support the circulation until myocardial recovery, as bridge to transplant, or as long-term destination therapy (DT) (4). Although LVAD recipients have excellent survival rates, postoperative adverse events can lead to impaired quality of life. The most common adverse events in the early and late periods after continuous flow LVAD implant are major infections (5). An infection rate of $9.1 \%$ in the first 3 months after LVAD implantation has been previously reported for pump-related percutaneous driveline infection (DLI) (6), that can lead to pain at the driveline exit site (DLES), an increase of medical costs, and even to stroke (7-9). Consequently, DLI is further the primary cause of readmission in LVAD patients (4). The development of DLI is multifactorial, with several reported risk factors such as increased body mass index (BMI) (10-13), history of diabetes mellitus (DM) (10), and an exposed velour (10, 14-16). The probability of developing a DLI seems to rise with the duration of LVAD support (17-19) and reaches a peak 6 months after implantation. This could be related to the increased activity of patients after hospital discharge (20) and the associated increase of trauma at the DLES, which was previously reported as one of the major initiators for DLI (21). Bending or torsion of the driveline is common during daily activity, e.g., changing of clothes, light exercises, or turning around while sleeping, which could lead to trauma at the DLES $(4,21)$ and rigid materials and large diameters of the driveline could exacerbate this problem. However, there is only limited knowledge about how driveline features such as diameter and stiffness of contemporary devices affect DLI occurrence. Therefore, this study aims to quantify and compare device-specific mechanical driveline properties of three LVADs with four different drivelines and to correlate them with DLI occurrence.

\section{MATERIALS AND METHODS}

\section{Meta-Analysis}

This meta-analysis is in accordance with the Preferred Reporting Items for Systematic Reviews and Meta-Analyses (PRISMA) statement. (22).

\section{Data Source and Search Strategy}

Two independent reviewers used the databases PubMed and SCOPUS in October 2021 with the search terms "Driveline Infection AND Left Ventricular Assist Devices," "Driveline Infection AND LVAD," "Driveline infection AND HeartMate 3," "Driveline infection AND HVAD," "Driveline infection AND HeartWare," and "Driveline Infection AND HeartMate II" to identify studies assessing DLI data of LVAD patients (Figure 1). Since the HVAD Carbothane driveline did not receive FDA

Abbreviations: ANOVA, analysis of variance; BMI, body mass index; DLES, driveline exit site; DLI, driveline infection; IQR, interquartile range; LVAD, left ventricular assist device; DM, diabetes mellitus; DT, destination therapy. approval until 2019 (23), and no studies were found in the database, an additional manual research was performed. The literature search was not limited to the strict PICO format, as this would likely have excluded relevant articles, particularly retrospective cohort studies without a control group.

\section{Study Selection and Data Extraction}

The outcomes of interest were either a numeric DLI rate at 6 months or a freedom from DLI Kaplan-Meier curve of at least one of the three devices and the sample size. Exclusion criteria included case reports, review articles, nonEnglish articles, records with wrong devices and records with unsuitable DLI rates (e.g., DLI rates stated as events per patient years). Extracted data included the study period, study design, the device, the DLI rate, the cohort's Interagency Registry for Mechanically Assisted Circulatory Support (INTERMACS) classification, DM, age, BMI, DT indication, gender, and implant technique characteristics. The WebPlotDigitizer (Version 4.4, Ankit Rohatgi, 2020) was used to extract the 6 months DLI rate from the Kaplan-Meier curve. A random-effects model was used and for each device type, the extracted DLI rates were weighted with the Schmidt-Hunter method depending on their sample size and used to calculate a mean weighted DLI rate. The evaluation, organization, and analysis of suitable literature sources were done using the software Review Manager (RevMan) (Version 5.4.1, The Cochrane Collaboration, 2020).

\section{Study Quality Assessment}

Studies were assessed for methodologic quality using the risk of bias tool described in the Cochrane Handbook for Systematic Reviews (24). This tool enables subjective assessment of bias across six domains, including selection, performance, attrition, detection, and reporting.

\section{Experimental Driveline Analysis Sample Selection}

An assortment of new and clinically used driveline samples without velour cover were analyzed. Eleven Pellethane HVAD (Medtronic Inc, Minneapolis, MN, USA), eleven Carbothane HVAD (Medtronic Inc), two HeartMate II (Abbott Inc, Chicago, IL, USA), and six HeartMate 3 (Abbott Inc) were used, and all measurements were repeated five times for each driveline specimen.

\section{Three-Point Bending Test}

An experimental three-point bending test (Figure 2A) was conducted, based on the standard EN ISO 178:2019 0801 (25) using a BOSE $囚$ LM1 ElectroForce test bench system (Bose Corp. $\mathrm{MN}$, USA) with an integrated displacement transducer. A 3Dprinted design with a support span of $30 \mathrm{~mm}$ was used, with the radii of the supports and the loading nose being $2.5 \mathrm{~mm}$. A $225 n$ load cell Type WMC-50-543 (Bose Corp. MN, USA) was mounted in line with the motor shaft. The measurement process was performed with the software WinTest $\AA$ (Version: 7.1.2014- 04.04, Bose Corp. MN, USA) allowing movements of the linear motor and simultaneous recording of time, load, and displacement. The drivelines were bent to a total displacement 


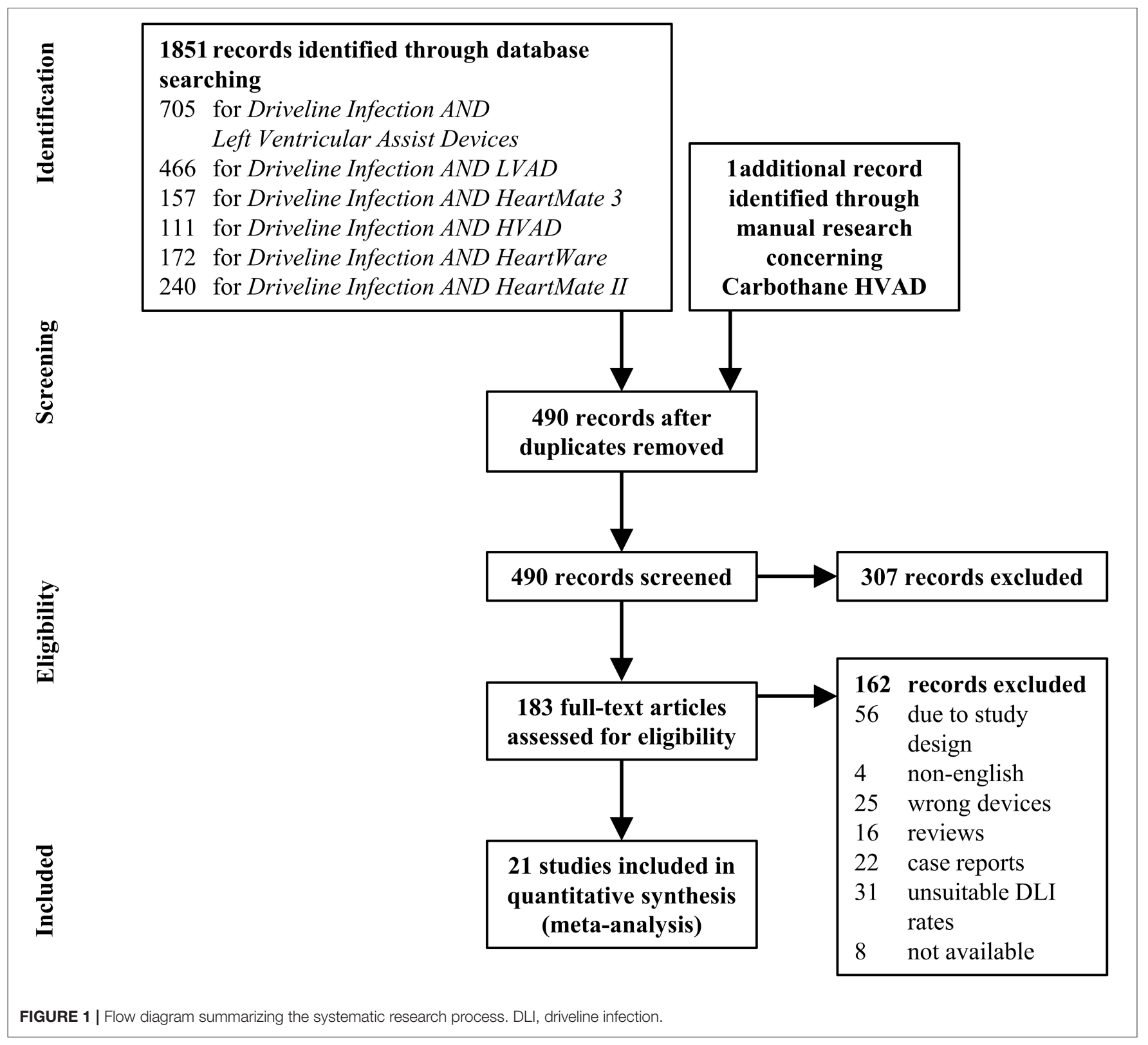

of $12 \mathrm{~mm}$ with a bending velocity of $1.5 \mathrm{~mm} / \mathrm{s}$ to measure the maximal bending force $\operatorname{Load}_{\max }$.

\section{Torsion Test}

The torsion test (Figure 2B) was modified from the standards EN 50289-3-10:2005 1101 (26) and EN ISO 25539- 2:2019 06 01 (27). The drivelines were clamped vertically into a custommade torsion testing apparatus with a free length of $12 \mathrm{~cm}$. An Arduino Uno R3 (Adafruit Industries, New York, USA) was used to operate a 42SHDC3025-24B stepper motor (Anet Technology Co., Ltd., Shenzhen, CHN) to twist the driveline $\left(720^{\circ}\right)$ with an angular velocity of $100 \%$ s. An iron bar was attached at the lower end of the driveline which was mounted in a tube as a duct and a lever arm with $10 \mathrm{~cm}$ was attached. When the stepper motor twisted the driveline, the lever arm pressed against a bar mounted on a RFS $\mathbb{R}$ ) $150 \mathrm{XY}$ sensor (Honigmann Industrielle Elektronik GmbH, Gevelsberg, DEU) to measure the maximal torque (Torque max ). The DS1103 PPC Controller Board and the software ControlDesk (Version: 5.0, 2013, dSPACE GmbH, Paderborn, DEU) were used for the simultaneous recording of time and torque.

\section{Statistical Analysis}

Descriptive statistics are reported as mean \pm standard deviation for normally distributed continuous variables and as median and interquartile range (IQR) for non-normally distributed values. Normal distribution was assessed by the Shapiro-Wilk test. Oneway analysis of variance (ANOVA) or Kruskal-Wallis tests were 
A

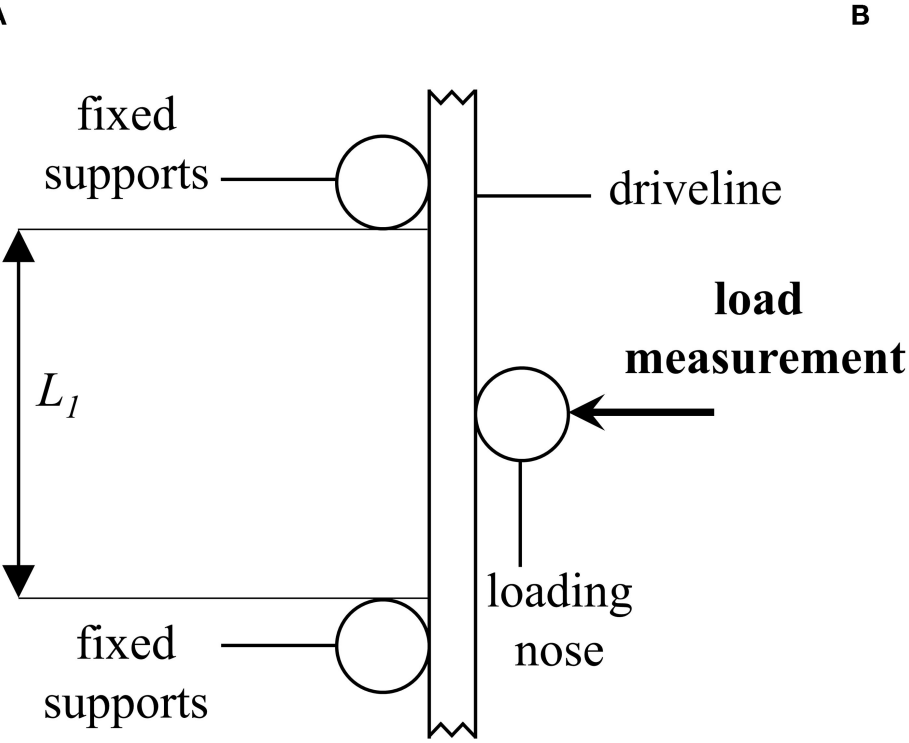

B

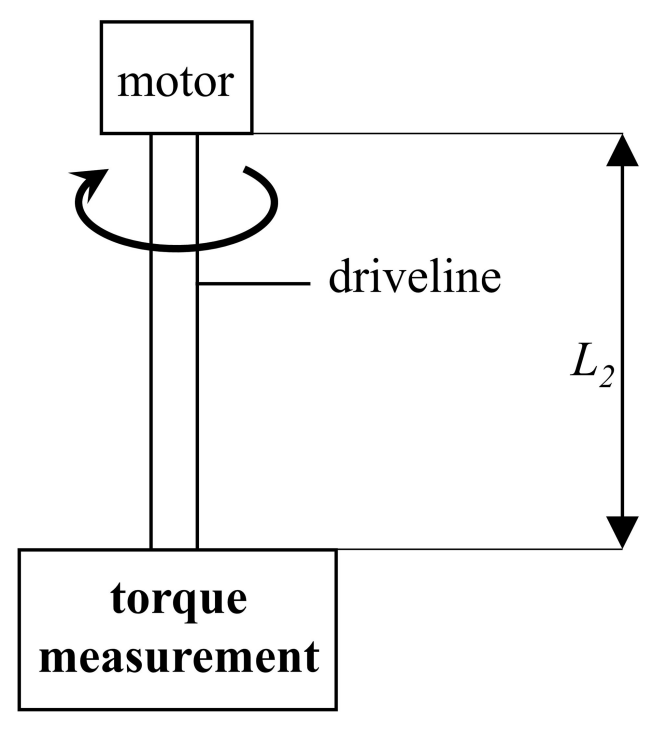

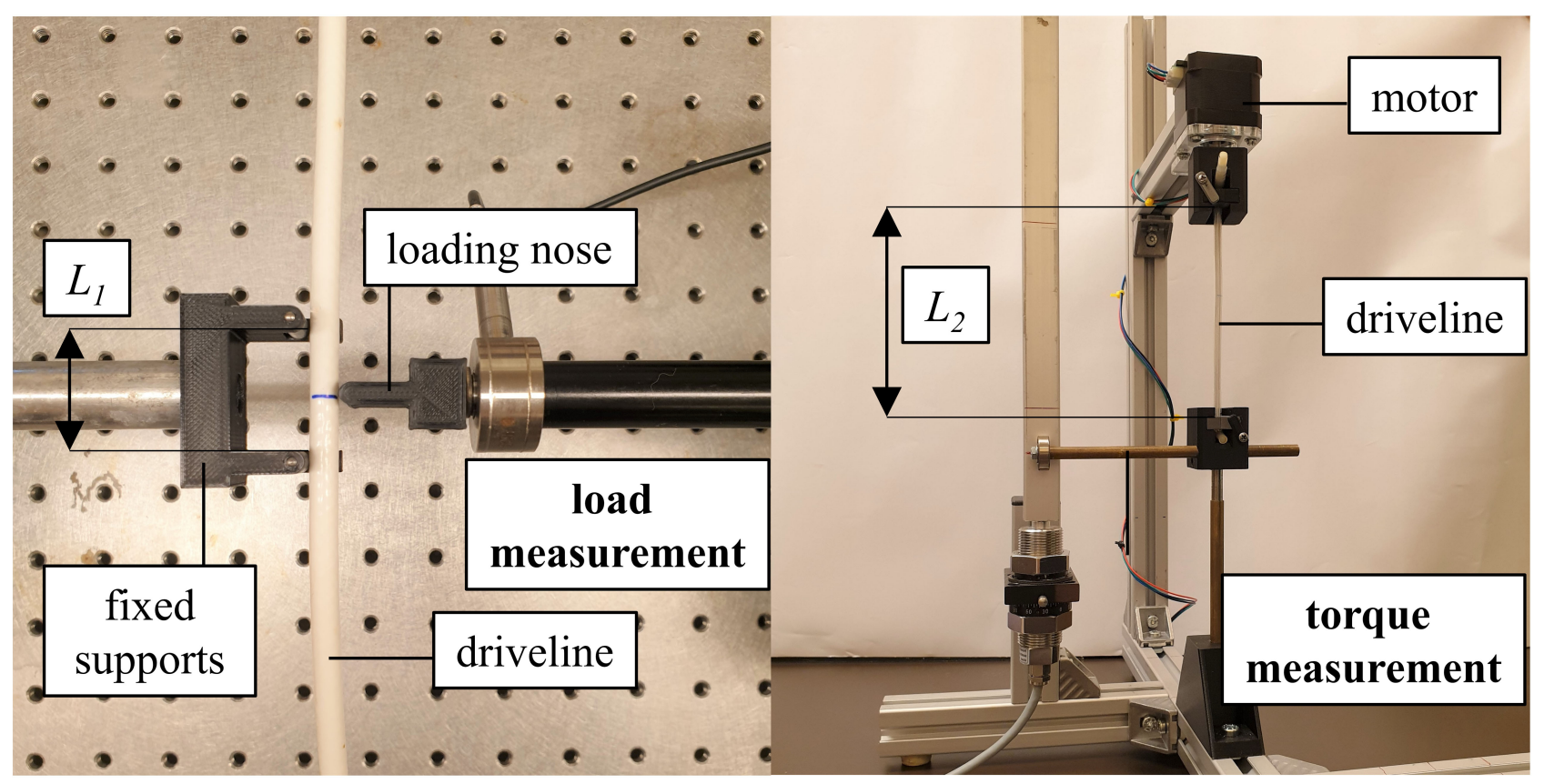

). $\left(L_{1}\right)$ support span, $\left(L_{2}\right)$ free length.

used to test continuous variables ( $\operatorname{Load}_{\max }$ and Torque $\max$ ) between the four driveline groups. When statistical significance was found $(p<0.05)$, post-hoc analyses were performed. Therefore, a Levene's test was used to check the homogeneity of variance with a significance level of $p<0.05$. If homogeneity of variance was present, a Bonferroni-test was performed, otherwise a Games Howell test for normally distributed groups was used. In both cases, the significance level was set to $p<0.05$. For non-normally distributed values, a pairwise comparison was performed with a Bonferroni correction, and the significance level was set at $p=0.0125$. Statistical analysis was performed by
SPSS for Windows Release 26.0.0 (SPSS Inc, Chicago, IL, USA) and MATLAB R2020a (The MathWorks Inc, Natick, MA, USA).

\section{RESULTS}

\section{Meta-Analysis}

Of the 490 full-text articles screened, $n=20$ articles fulfilled the inclusion criteria and reported DLI rates at 6 months following LVAD implantation in one or more of the included device types (Figure 1). Manual search on DLI rates of patients supported with the Carbothane HVAD driveline revealed $n=1$ abstract. 


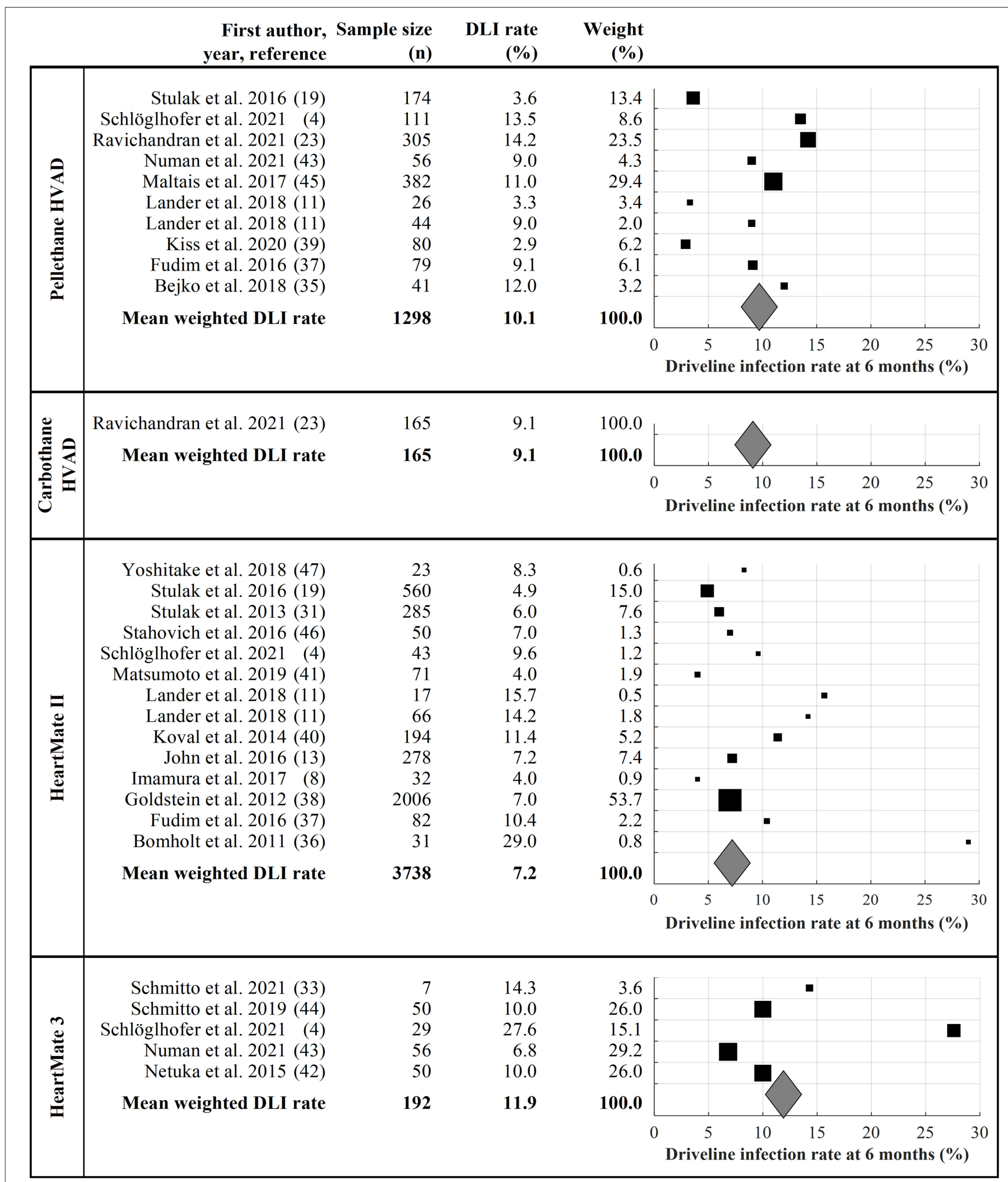

FIGURE 3 | Summary of all included and analyzed studies. The black marks represent the DLI of the study, the size of the black marks refers to the number of included patients. The mean weighted driveline infection rate for each device is represented by gray diamonds. DLI, driveline infection rate. 
TABLE 1 | Summary of the mechanical features of the analyzed drivelines.

\begin{tabular}{lccc}
\hline & Load $_{\max }$ [N] & Torque $_{\max }[\mathrm{mNm}]$ & Diameter [mm] \\
\hline Pellethane HVAD & $15.55 \pm 0.14$ & $94.62(3.89)$ & $4.8(0.0)$ \\
Carbothane HVAD & $8.50 \pm 0.08$ & $46.06(3.78)$ & $4.8(0.0)$ \\
HeartMate II & $4.52 \pm 0.19$ & $41.44(12.61)$ & $6.0(0.0)$ \\
HeartMate 3 & $11.08 \pm 0.30$ & $95.63(26.6)$ & $6.6(0.58)$ \\
\hline
\end{tabular}

Values are either presented as mean \pm standard deviation for normally distributed groups or as median (interquartile range) for non-normally distributed groups. Load max $_{\text {, maximal }}$ bending force; Torque max, maximal torque.

In total, 5,393 patients were included in the final meta-analysis. The most studies $(n=14)$ and included patients $(n=3738)$ were identified for the HeartMate II. The mean weighted DLI rates ranged from $7.2 \%$ (HeartMate II) to $11.9 \%$ (HeartMate 3 ). The final 21 articles, including the DLI rate after 6 months for each study and the mean weighted DLI rate for each driveline type are summarized in Figure 3. The overall mean weighted DLI rate including all studies was $8.1 \%$. Of the included studies with reported patient characteristics, INTERMACS Class 1 ranged from 0 to $41.0 \%$, age was between $38 \pm 13$ and $62.4 \pm 8.3$, BMI ranged from $20.4 \pm 3.5$ and $29.7 \pm 6.23,13.0 \%$ to $43.8 \%$ suffered from DM, $11 \%$ to $100 \%$ received their LVAD as DT, and $65.4 \%$ to 93\% were male (see Supplementary Table 1).

Assessments of study quality and risk of bias are summarized in Supplementary Table 2. In the fast majority of studies, a low risk for performance (85.7\%), detection (100\%) and reporting $(90.5 \%)$ bias was found. Moderate selection bias was more common $(23.8 \%)$, whereas the attrition bias was rated as low $(66.6 \%)$ or unclear $(23.8 \%)$ in most studies.

\section{Experimental Driveline Analysis}

In total, 30 driveline samples were analyzed and Table 1 summarizes their mechanical features. Among the four observed driveline types, Carbothane HVAD and Pellethane HVAD had the smallest diameter, with $4.8(0.0) \mathrm{mm}$. The least rigid driveline in the three-point bending test was the HeartMate II ( $\operatorname{Load}_{\max }$ $=4.27 \pm 0.07 \mathrm{~N}$ ), whereas the Pellethane HVAD driveline had a significantly higher $\operatorname{Load}_{\text {max }}=13.56 \pm 0.08 n(p<0.001)$. The stiffness of each driveline type is shown in Figure 4A. Significant differences $(p<0.001)$ were found between all groups. In the torsion tests (Figure 4B), the HeartMate II driveline had the lowest Torque $\max [41.44(12.61) \mathrm{mNm}$ ] and the HeartMate 3 driveline had the highest Torque $\max [95.63$ (26.60) $\mathrm{mNm}$ ]. Further, the HeartMate 3 driveline Torque $_{\max }$ was significantly higher $(p<0.0125)$ compared to the Carbothane HVAD and the HeartMate II drivelines. Comparable results were found between the Carbothane HVAD and the HeartMate II $(p=0.95)$ as well as between the Pellethane HVAD and the HeartMate 3 drivelines $(p=0.69)$.

\section{Relationship of DLI Rates and Driveline Features}

Figure 5 summarizes the relationships between the mechanical characteristics of the four different drivelines from the ex-vivo experimental study and the mean weighted DLI rates at 6 months. No relevant association between the mean weighted DLI rate and the driveline diameter (Figure 5A) or the Load $_{\max }$ of the three-point bending test (Figure 5B) was found, respectively. There was an apparent relationship between Torque $\max$ of the torsion test and the mean weighted DLI rate (Figure 5C); The HeartMate II driveline had the lowest Torque $\max$ and lowest DLI rate, whereas the HeartMate 3 driveline had the highest Torque $_{\max }$ and highest DLI rate.

\section{DISCUSSION}

DLI is one of the most common adverse events in the early and late phases after LVAD implantation (1). The development of DLI is multifactorial, with several reported non-modifiable risk factors like DM $(10)$, age $(12,16,28)$ or exposed velour $(10,14-$ 16), and, on the other hand, modifiable risk factors (29), such as BMI (10-13), patient lifestyle and activity following hospital, discharge and the associated increase in trauma at the DLES (21). To the best of our knowledge, only one other study has reported the correlation between mechanical driveline features and DLI rates of LVAD-patients (8), but data for contemporary devices are missing. Therefore, the aim of this study was to quantify and compare device-specific driveline characteristics of the HVAD, HeartMate II, and HeartMate 3 as an additional modifiable risk factor associated with DLI, both in-vivo and ex-vivo (Figure 6).

As previously reported (20), DLI peaks 6 months after LVAD implantation. In this meta-analysis, we found a DLI rate of $8.1 \%$ at 6 months, making DLI one of the major adverse events after LVAD implantation. The mean weighted DLI rate was highest with the HeartMate 3 (11.9\%), compared with the Pellethane HVAD (10.1\%), the Carbothane HVAD (9.1\%), and the HeartMate II (7.2\%). Therefore, regardless of patient demographics and center-specific DLES care protocols, the HeartMate II may have positive mechanical driveline features compared to other commercially available LVADs. The approaches for the development of the four contemporary LVAD drivelines investigated in this study are diverse, and different materials are used. Whereas the HeartMate II driveline consists of a soft silicone-based outer layer enveloping an inner jacket made of polyurethane wrapped around a fiber core made of polyethylene (30), the HeartMate 3 driveline has a siliconebased outer layer wrapped around a fiber layer of braided aramid enveloping a polytetrafluoroethylene layer (4). HVAD drivelines consist of an inner silicone lumen enveloped by either Pellethane ${ }^{\circledR}$ or with the new Carbothane ${ }^{\circledR}$ design (23).

Consequently, our ex-vivo experimental study showed significant differences in driveline stiffness between all devices $(p<0.001)$ as assessed by the $\operatorname{Load}_{\max }$ of the three-point bending test (Figure 4A). Even though there was no obvious relationship between Load $_{\max }$ and the mean weighted DLI rate, the HeartMate 3 driveline with the largest diameter had the highest DLI rate (Figure 5B). This is in accordance with the findings of Imamura et al., who reported that the HeartMate II driveline had only $20-25 \%$ of stiffness and a smaller diameter compared to other devices (EVAHEART, and DuraHeart) and the 

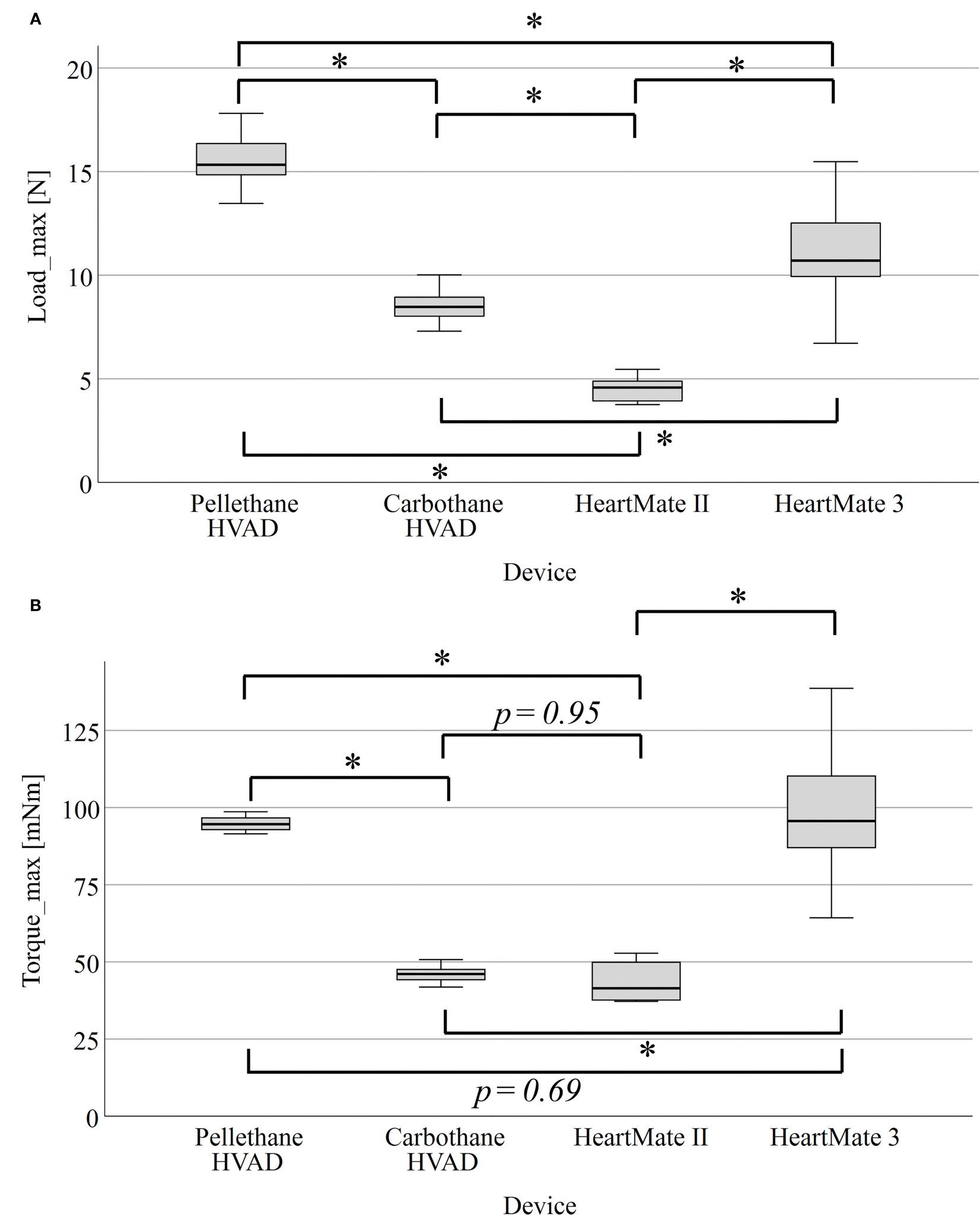

FIGURE 4 | Boxplots of Load ${ }_{\max }$ of the three-point bending test of four different driveline types (A) and Torque $\max$ of the torsion tests (B). ${ }^{*} p<0.001$. Loadmax, maximal bending force; Torque max maximal torque. 
A

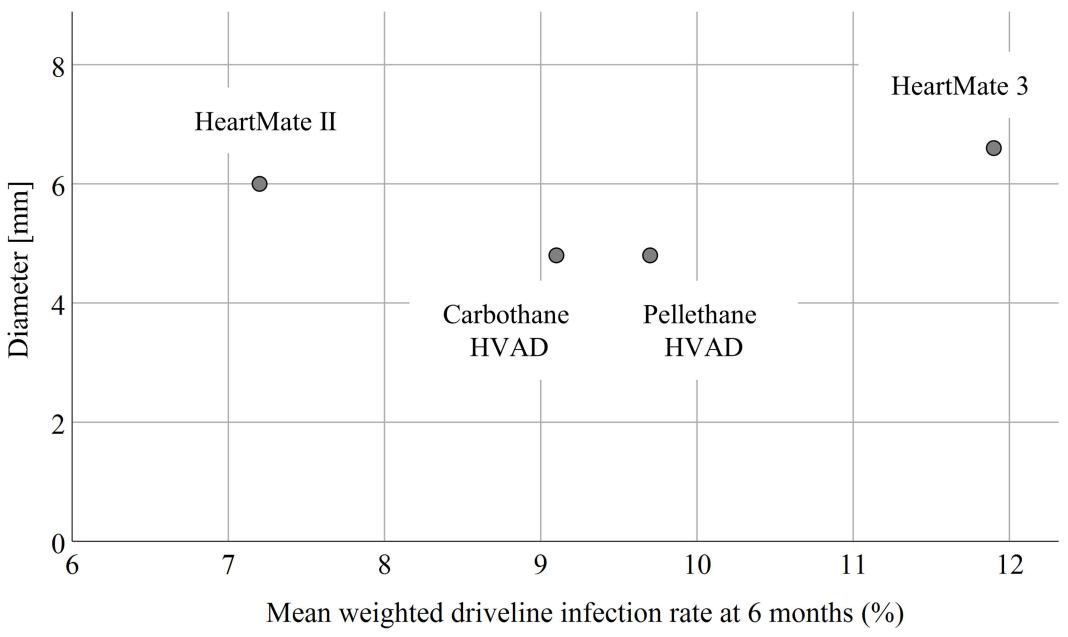

B

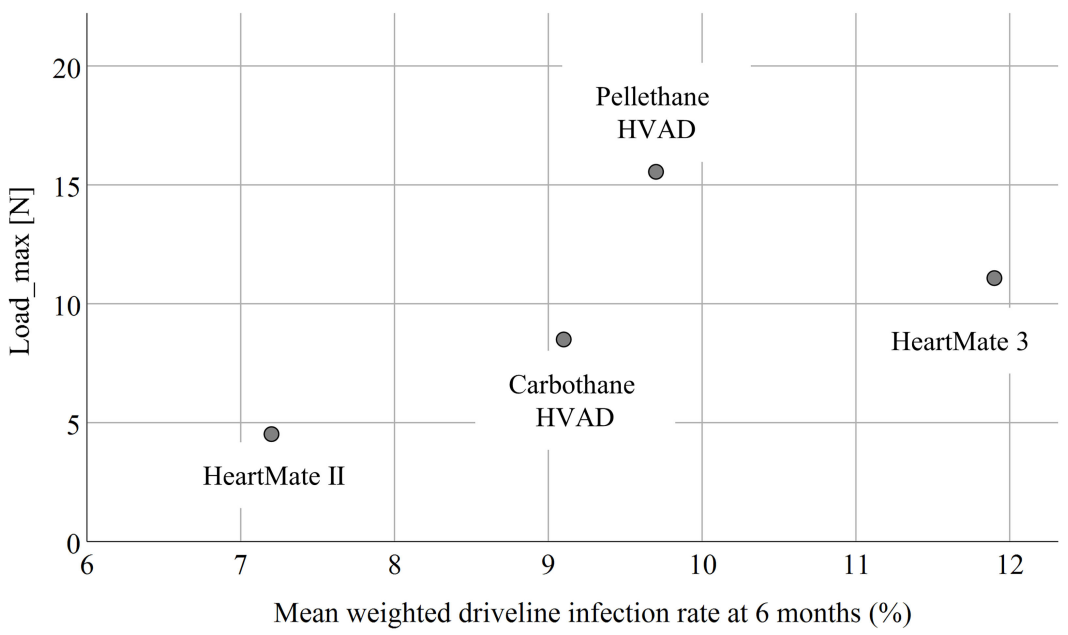

C

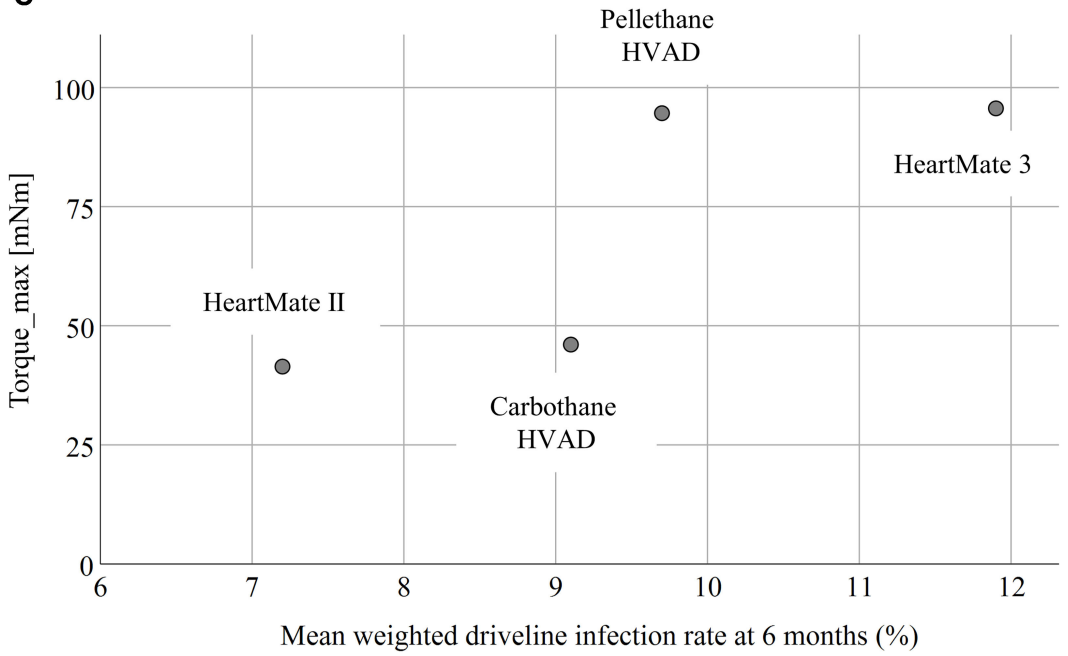

FIGURE 5 | Correlation between the diameter (A), Load $\max$ (B), and Torque $\max (\mathbf{C})$ and the mean weighted driveline infection rate at six months. Load max $_{\text {, maximal }}$ bending force; Torque max , maximal torque. 

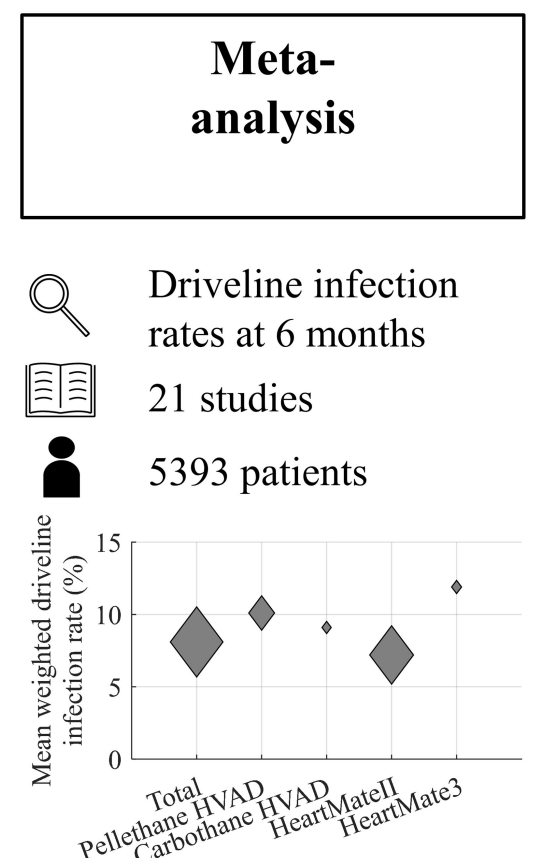

\section{K E Y F I N D I N G S}

Mechanical driveline features are an additional modifiable risk factor for driveline infection in left ventricular assist device patients.

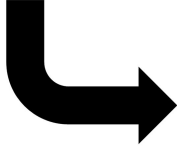

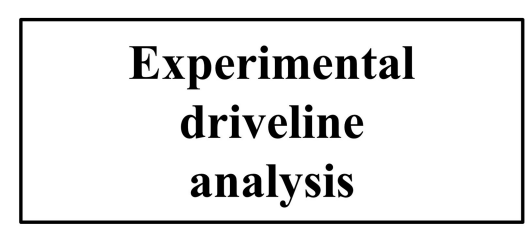

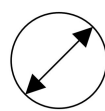

Diameter

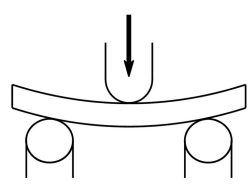

Three-point

bending test

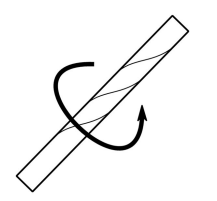

\section{Torsion test}

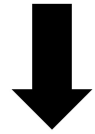

Flexible and thin drivelines

Flexible and thin d
preferred

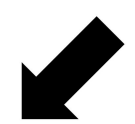

\section{Driveline fixation close to the driveline exit site}
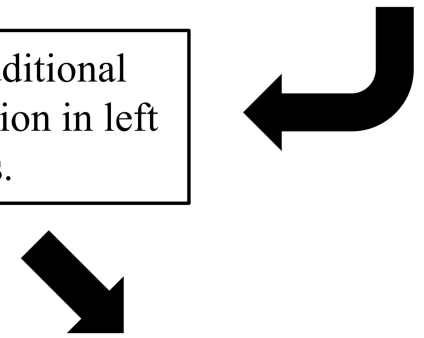

\section{New HeartMate 3 dressing methods may be required}

\section{L I N I C A L I M P L I C A T I O N S}

FIGURE 6 | Graphical abstract summarizing the in-vivo and ex-vivo analysis of mechanical driveline features as a risk factor for driveline infection.

highest DLI-free rate among those three devices (8). However, the key finding of this study was the hypothesis-generating apparent relationship between higher Torque max $_{\text {of }}$ of the torsion test and the increased DLI rates (Figure 5C). Therefore, this parameter seems to be a crucial marker for further technical improvements, as driveline torsion is a frequent event in the daily life of LVAD patients, potentially exerting additional force on the DLES and thus leading to trauma-induced DLI as the adherent interface between the velour of the internal part of the driveline and the patient's tissues is critical for the protection against entry of microorganisms and subsequent infection (31). These findings could be relevant to clinical practice, as mechanical features are a modifiable risk factor and exploring more flexible and thinner drivelines would be a simple means to prevent
DLI. Based on our ex-vivo results and in relation to the clinical DLI rates resulting from the meta-analysis, the most important feature of a LVAD driveline seems to be high flexibility (in terms of low Torque max $)$, followed by low stiffness $\left(\operatorname{Load}_{\max }\right)$, and minimal thickness (diameter). Although the Medtronic HVAD was recently withdrawn from the market, the development of the new Carbothane driveline appears to be the first step in the multi-faceted strategies to reduce DLI and, by extension, a risk factor for one of the most feared and devastating complications during LVAD support-stroke (7-9). Since the relationship between driveline mechanical properties and DLI rates appears moderate, driveline features are certainly not the "only" risk factor, but are definitely a previously unknown additional factor in DLI development. Therefore, the technical improvement of 
the mechanical properties of drivelines or even the elimination of them by transcutaneous energy transfer systems (32) should be a high priority in the future development of LVADs. In addition, the development of transcutaneous energy transfer systems will make disappear the need for periodic driveline repairs or for exchanging the HeartMate 3 modular cable-which was necessary in $50 \%$ of long-term patients as their active lifestyles caused the cable to deteriorate (33). Thus, the HeartMate 3 modular cable is both a curse and a blessing - the connector enables these necessary driveline exchanges, but this design feature may also be the reason for the higher DLI rates as the rigid modular connector might apply additional traction on the DLES compared to the other devices. Therefore, the results of our study lead to the hypothesis that the overall HeartMate 3 driveline design, including modular connector, is unfavorable, but the findings contrast with the MOMENTUM 3 final report (34), which found no significant but numerically higher 2-year DLI rates with HeartMate 3 (23.3\%) vs. HeartMate II (19.4\%).

Finally, it should be mentioned that with the HeartMate 3 as the only commercially available LVAD, new DLES dressing methods may be required, including additional binders or anchoring devices (4), e.g., to fix the driveline and the modular connector in a U-shape directly as close as possible at the DLES as well as the rigid connector on the skin to minimize driveline movement and trauma to prevent DLI. The design of nextgeneration LVAD peripherals should therefore possibly have a combination of external helix pump cable from the controller to the modular connector to absorb additional forces, followed by the most flexible and thin driveline possible to the DLES and implanted pump.

\section{Limitations}

This study has limitations that should be considered when interpreting the results. The meta-analysis was limited to 21 articles (35-47), including only one multicenter study that reported DLI rates of the Carbothane HVAD. Differences in study design, patient characteristics and selection, and center specific DLES care protocols might vary between centers, so we cannot

\section{REFERENCES}

1. Piazza V, Montalto A, Amarelli C, Loforte A, Musumeci F. Physiopathology and fate of end-stage chf in the era of MCS. In: Montalto A, Loforte A, Musumeci F, Krabatsch T, Slaughter MS, Editors. Mechanical Circulatory Support in End-Stage Heart Failure. Cham: Springer International Publishing. (2017) p. 13-24.

2. Reyes G, Badia S. Indications for ventricle assist devices. In: Shuhaiber JH, Editor. Ventricular Assist Devices. Rijeka, Croatia: InTech. (2011) p. 3-24.

3. Pagani FD. Understanding the principles of continuous flow rotary left ventricular assist devices. In: Kirklin JK, Rogers JG, editors. Mechanical Circulatory Support: A Companion to Braunwald's Heart Disease. 2nd ed. Maryland Heights: Elsevier Inc. (2019) p. 71-81.

4. Schlöglhofer T, Michalovics P, Riebandt J, Angleitner P, Stoiber M, Laufer G, et al. Left ventricular assist device driveline infections in three contemporary devices. Artificial Organs. (2021) 45:464-72. doi: 10.1111/aor.13843

5. Molina EJ, Shah P, Kiernan MS, Cornwell WK, Copeland H, Takeda K et al. The society of thoracic surgeons intermacs 2020 annual report. Ann Thorac Surg. (2021) 111:778-92. doi: 10.1016/j.athoracsur.2020.12.038 exclude previously reported factors affecting the occurrence of DLI at all. In addition, experimental mechanical testing was limited to a rather modest number of drivelines $(n=30)$, including new and clinically used ones without velour cover or modular driveline connectors (HeartMate 3 ). The effects of chemical and physical aging of drivelines used in-vivo on stiffness were not investigated in this study.

\section{CONCLUSION}

Device-specific mechanical features of the driveline are an additional modifiable risk factor for the development of DLI and may influence clinical outcomes of LVAD patients.

\section{DATA AVAILABILITY STATEMENT}

The raw data supporting the conclusions of this article will be made available by the authors, without undue reservation.

\section{AUTHOR CONTRIBUTIONS}

MK, MS, and TS developed the concept and design, performed the statistical analysis, and funding was secured by DZ, GL, HS, and TS. MK and TS drafted the article. MK, A-KS, JR, DW, and $\mathrm{CM}$ collected the data. All authors performed critical revision of the article and approved the final version.

\section{ACKNOWLEDGMENTS}

We would like to thank Abbott Inc. and Medtronic Inc. for providing driveline samples.

\section{SUPPLEMENTARY MATERIAL}

The Supplementary Material for this article can be found online at: https://www.frontiersin.org/articles/10.3389/fcvm. 2021.784208/full\#supplementary-material
6. Hannan MM, Xie R, Cowger J, Schueler S, By T de, Dipchand AI et al. Epidemiology of infection in mechanical circulatory support: a global analysis from the ishlt mechanically assisted circulatory support registry. J Heart Lung Transplant. (2019) 38:364-73. doi: 10.1016/j.healun.2019. 01.007

7. Frontera JA, Starling R, Cho S-M, Nowacki AS, Uchino K, Hussain MS et al. Risk factors, mortality, and timing of ischemic and hemorrhagic stroke with left ventricular assist devices. J Heart Lung Transplant. (2017) 36:673-83. doi: 10.1016/j.healun.2016.12.010

8. Imamura T, Murasawa T, Kawasaki H, Kashiwa K, Kinoshita O, Nawata $\mathrm{K}$ et al. Correlation Between Driveline Features and Driveline Infection in Left Ventricular Assist Device Selection. J Artific Organs. (2017) 20:34-41. doi: 10.1007/s10047-016-0923-8

9. Cho S-M, Moazami N, Katz S, Bhimraj A, Shrestha NK, Frontera JA. Stroke risk following infection in patients with continuous-flow left ventricular assist device. Neurocrit Care. (2019) 31:72-80. doi: 10.1007/s12028-018-0662-1

10. Donahey EE, Polly DM, Vega JD, Lyon M, Butler J, Nguyen D et al. Multidrugresistant organism infections in patients with left ventricular assist devices. Texas Heart Instit J. (2015) 42:522-7. doi: 10.14503/THIJ-14-4612 
11. Lander M, Kunz N, Dunn E, Lockard K, O'Shea G, Weaver S et al. Substantial reduction in driveline infection rates with the modification of driveline dressing protocols. J Heart Lung Transplant. (2018) 35:746-52. doi: 10.1016/j.cardfail.2018.07.464

12. John R, Aaronson KD, Pae WE, Acker MA, Hathaway DR, Najarian KB et al. Drive-line infections and sepsis in patients receiving the HVAD system as a left ventricular assist device. J Heart Lung Transplant. (2014) 33:1066-73. doi: 10.1016/j.healun.2014.05.010

13. John R, Holley CT, Eckman P, Roy SS, Cogswell R, Harvey L et al. A decade of experience with continuous-flow left ventricular assist devices. Semin Thoracic Cardiovascul Surg. (2016) 28:363-75. doi: 10.1053/j.semtcvs.2016. 05.013

14. Singh A, Russo MJ, Valeroso TB, Anderson AS, Rich JD, Jeevanandam V et al. Modified heartmate ii driveline externalization technique significantly decreases incidence of infection and improves long-term survival. ASAIO J. (2014) 60:613-6. doi: 10.1097/MAT.0000000000000121

15. McCandless SP, Ledford ID, Mason NO, Alharethi R, Rasmusson BY, Budge $\mathrm{D}$ et al. Comparing velour versus silicone interfaces at the driveline exit site of heartmate ii devices: infection rates, histopathology, and ultrastructural aspects. Cardiovascul Pathol. (2015) 24:71-5. doi: 10.1016/j.carpath.2014.07.011

16. Dean D, Kallel F, Ewald GA, Tatooles A, Sheridan BC, Brewer RJ et al. Reduction in driveline infection rates: results from the heartmate ii multicenter driveline silicone skin interface (ssi) registry. J Heart Lung Transplant. (2015) 34:781-9. doi: 10.1016/j.healun.2014.11.021

17. Kusne S, Staley L, Arabia F, Snydman DR. Prevention and infection management in mechanical circulatory support device recipients. Clinic Infect Dis. (2017) 64:222-8. doi: 10.1093/cid/ciw698

18. Sharma V, Deo SV, Stulak JM, Baddour LM, Mehra K, Joyce LD. Driveline infections in left ventricular assist devices: implications for destination therapy. Ann Thoracic Surg. (2012) 94:1381-6. doi: 10.1016/j.athoracsur.2012.05.074

19. Stulak JM, Davis ME, Haglund N, Dunlay S, Cowger J, Shah $p$ et al. Adverse events in contemporary continuous-flow left ventricular assist devices: a multi-institutional comparison shows significant differences. $J$ Thoracic Cardiovascul Surg. (2016) 151:177-89. doi: 10.1016/j.jtcvs.2015. 09.100

20. Qu Y, Peleg AY, McGiffin D. Ventricular assist device-specific infections. J Clinic Med. (2021) 10:453. doi: 10.3390/jcm10030453

21. Zierer A, Melby SJ, Voeller RK, Guthrie TJ, Ewald GA, Shelton K et al. Late-onset driveline infections: the achilles' heel of prolonged left ventricular assist device support. Annal Thoracic Surg. (2007) 84:515-20. doi: 10.1016/j.athoracsur.2007.03.085

22. Moher D, Liberati A, Tetzlaff J, Altman DG. Preferred reporting items for systematic reviews and meta-analyses: the prisma statement. PLoS Med. (2009) 6:E100(0097). doi: 10.1371/journal.pmed.1000097

23. Ravichandran AK, Cowger JA, Feller ED, Mahr C, Hiesinger W, Klein L et al. An analysis of driveline infections with left ventricular assist devices utilizing carbothane versus pellethane driveline sheaths. J Heart Lung Transplant. (2021) 40:434-435. doi: 10.1016/j.healun.2021.01.1213

24. Higgins JPT, Thomas J, Chandler J, Cumpston M, Li T, Page MJ, et al. cochrane handbook for systematic reviews of interventions version 6.2 (Updated February 2021): Cochrane (2021). Available online at: www.Training.Cochrane.org/Handbook.

25. Austrian Standards International. OENORM EN ISO 178:2019 0801 Kunststoffe-Bestimmung der Biegeeigenschaften.

26. Austrian Standards International. OEVE/OENORM EN 50289-3-10:2005 1101 Kommunikationskabel-Spezifikationen für Prüfverfahren. Teil 3-10: Mechanische Prüfverfahren-Torsion und Verdrillung.

27. Austrian Standards International. OENORM EN ISO 25539-2:2013 0601. Kardiovaskuläre Implantate-Endovaskuläre Implantate. Teil 2: Gefäßstents.

28. Imamura $T$, Kinugawa $K$, Nitta $D$, Inaba $T$, Maki $H$, Hatano $M$ et al. Readmission due to driveline infection can be predicted by new score by using serum albumin and body mass index during long-term left ventricular assist device support. J Artific Organs. (2015) 18:120-7. doi: 10.1007/s10047-015-0816-2

29. Bernhardt AM, Schlöglhofer T, Lauenroth V, Mueller F, Mueller M, Schoede A et al. Prevention and early treatment of driveline infections in ventricular assist device patients-the destine staging proposal and the first standard of care protocol. J Critic Care. (2020) 56:106-12. doi: 10.1016/j.jcrc.2019. 12.014

30. Flint KM, Brieke A, Cornwell WK, Pierce C, Cleveland JC, Pal JD. Heartmate ii system controller failure presenting as driveline fault with repeated pump stoppages. Circ: Heart Failure. (2019) 12:E00(5738). doi: 10.1161/CIRCHEARTFAILURE.118.005738

31. Stulak JM, Maltais S, Cowger J, Joyce LD, Daly RC, Park SJ et al. Prevention of percutaneous driveline infection after left ventricular assist device implantation: prophylactic antibiotics are not necessary. ASAIO J. (2013) 59:570-4. doi: 10.1097/MAT.0b013e3182a $9 \mathrm{e} 2 \mathrm{a} 5$

32. Pya Y, Maly J, Bekbossynova M, Salov R, Schueler S, Meyns B et al. First human use of a wireless coplanar energy transfer coupled with a continuousflow left ventricular assist device. J Heart Lung Transplant. (2019) 38:339-43. doi: 10.1016/j.healun.2019.01.1316

33. Schmitto JD, Mariani S, Li T, Dogan G, Hanke JS, Bara C et al. Five-year outcomes of patients supported with heartmate 3: a single-centre experience. Euro J Cardio-Thoracic Surg. (2021) 59:1155-63. doi: 10.1093/ejcts/ez ab018

34. Mehra MR, Naka Y, Uriel N, Goldstein DJ, Cleveland JC, Colombo PC et al. A fully magnetically levitated circulatory pump for advanced heart failure. NE JMed. (2017) 376:440-50. doi: 10.1056/NEJMoa1610426

35. Bejko J, Toto F, Gregori D, Gerosa G, Bottio T. Left ventricle assist devices and driveline's infection incidence: a single-centre experience. J Artific Organs. (2018) 21:52-60. doi: 10.1007/s10047-017-0997-y

36. Bomholt T, Moser C, Sander K, Boesgaard S, Køber L, Olsen PS et al. Driveline infections in patients supported with a heartmate ii: incidence, aetiology and outcome. Scand Cardiovascul J. (2011) 45:273-8. doi: 10.3109/14017431.2011.577236

37. Fudim M, Brown CL, Davis ME, Djunaidi M, Danter MR, Harrell FE et al. Driveline infection risk with utilization of a temporary external anchoring suture after implantation of a left ventricular assist device. ASAIO J. (2016) 62:291-6. doi: 10.1097/MAT.0000000000000346

38. Goldstein DJ, Naftel D, Holman W, Bellumkonda L, Pamboukian SV, Pagani FD et al. Continuous-?ow devices and percutaneous site infections: clinical outcomes. J Heart Lung Transplant. (2012) 31:1151-7. doi: 10.1016/j.healun.2012.05.004

39. Kiss J, Stark C, Nykänen A, Lemström K. Outcome of patients receiving a continuous flow left ventricular assist device-a retrospective single center study. Scand Cardiovascul J. (2020) 54:212-9. doi: 10.1080/14017431.2020.1751264

40. Koval CE, Thuita L, Moazami N, Blackstone E. Evolution and impact of drive-line infection in a large cohort of continuous-flow ventricular assist device recipients. J Heart Lung Transplant. (2014) 33:1164-72. doi: 10.1016/j.healun.2014.05.011

41. Matsumoto Y, Fukushima S, Shimahara Y, Yamashita K, Kawamoto N, Kuroda $\mathrm{K}$ et al. Driveline angle is crucial for preventing driveline infection in patients with heartmate ii device. J Artific Organs. (2019) 22:37-43. doi: $10.1007 / \mathrm{s} 10047-018-1074-\mathrm{x}$

42. Netuka I, Sood P, Pya Y, Zimpfer D, Krabatsch T, Garbade J et al. Fully magnetically levitated left ventricular assist system for treating advanced hf: a multicenter study. J Am Coll Cardiol. (2015) 66:2579-89. doi: 10.1016/j.jacc.2015.09.083

43. Numan L, Ramjankhan FZ, Oberski DL, Oerlemans MIFJ, Aarts E, Gianoli $\mathrm{M}$ et al. Propensity score-based analysis of long-term outcome of patients on heartware and heartmate 3 left ventricular assist device support. ESC Heart Failure. (2021) 8:1596-603. doi: 10.1002/ehf2.13267

44. Schmitto JD, Pya Y, Zimpfer D, Krabatsch T, Garbade J, Rao V et al. Long-term evaluation of a fully magnetically levitated circulatory support device for advanced heart failure-two-year results from the heartmate 3 ce mark study. Euro J Heart Failure. (2019) 21:90-7. doi: 10.1002/ejh f. 1284

45. Maltais S, Aaronson KD, Teuteberg JJ, Slaughter MS, Najjar SS, Jeevanandam $\mathrm{V}$ et al. Adverse event rates change favorably over time for patients bridged with the heartware left ventricular assist device. ASAIO J. (2017) 63:745-51. doi: 10.1097/MAT.00000000000 00585 
46. Stahovich M, Sundareswaran KS, Fox S, Hallinan W, Blood P, Chen L et al. Reduce driveline trauma through stabilization and exit site management: 30 days feasibility results from the multicenter resist study. ASAIO J. (2016) 62:240-5. doi: 10.1097/MAT.0000000000000374

47. Yoshitake S, Kinoshita O, Nawata K, Hoshino Y, Itoda Y, Kimura M et al. Novel driveline route for prevention from driveline infection: triple tunnel method. J Cardiolo. (2018) 72:363-6. doi: 10.1016/j.jjcc.2018. 04.003

Conflict of Interest: TS has served as a consultant and advisor for Medtronic Inc. and Abbott Inc. and has received research grants from Medtronic Inc. and Abbott Inc. DW has served as a proctor and advisor for Medtronic Inc. and Abbott Inc. HS has served as an advisor for Medtronic Inc. and has received research grants from Medtronic Inc. DZ has served as a proctor, advisor, and speaker for Medtronic Inc., Abbott Inc., Berlin Heart, Edwards, Abiomed, and has received research and travel grants from Medtronic Inc. and Abbott Inc.
The remaining authors declare that the research was conducted in the absence of any commercial or financial relationships that could be construed as a potential conflict of interest.

Publisher's Note: All claims expressed in this article are solely those of the authors and do not necessarily represent those of their affiliated organizations, or those of the publisher, the editors and the reviewers. Any product that may be evaluated in this article, or claim that may be made by its manufacturer, is not guaranteed or endorsed by the publisher.

Copyright (c) 2021 Kranzl, Stoiber, Schaefer, Riebandt, Wiedemann, Marko, Laufer, Zimpfer, Schima and Schlöglhofer. This is an open-access article distributed under the terms of the Creative Commons Attribution License (CC BY). The use, distribution or reproduction in other forums is permitted, provided the original author(s) and the copyright owner(s) are credited and that the original publication in this journal is cited, in accordance with accepted academic practice. No use, distribution or reproduction is permitted which does not comply with these terms. 